\title{
On the spatial-temporal development of synthetic turbulent boundary layer on a serrated trailing edge
}

\author{
A. Juknevicius ${ }^{1}$ and T.P. Chong ${ }^{2}$ \\ Department of Mechanical and Aerospace Engineering, Brunel University London, \\ Uxbridge, UB8 $3 P H$
}

\begin{abstract}
This paper presents results from an experimental study to investigate the flow mechanism that enables the add-on saw-tooth serrations to achieve the reduction of broadband trailing edge noise produced by a NACA 0008 aerofoil. The current work traces the temporal-spatial development of an artificially generated synthetic turbulent boundary layer (SBL) on a straight and serrated aerofoil trailing edge. The experiment was performed at $\mathrm{Re}=0.96 \times 10^{5}$ based on the aerofoil chord. Temporal variations in the acoustic pressure radiated as a result of the flow interaction between the aerofoil trailing edge and the SBL were studied by conducting a wavelet analysis of the far-field noise measurement data. The results successfully capture a short duration variation in the radiated acoustic pressure associated with the passing of the SBL over the trailing edges. Comparison of the results obtained for the straight and serrated trailing edges identifies noise reduction over a short time domain that is exactly linked to a reduced level of wallnormal turbulence intensity close to the surface of trailing edge serrations. A distinct high momentum vortical structure is formed in the near wake of serrations at a later stage of the SBL. This secondary flow is responsible for the reduction of the fluctuations in the wall-normal velocity component at the serration, as well as the increase of the anisotropic level of the turbulence at the serration. As a result, turbulence scattering at the oblique edges is weakened, leading to the reduction of the radiated noise. The results presented in this paper provide a new outlook on the turbulence interaction with trailing edge serrations and the associated trailing edge broadband noise generation.
\end{abstract}

\section{Nomenclature}

$\begin{array}{ll}2 h & =\text { serration amplitude } \\ a & =\text { hot-wire overheat ratio } \\ b & =\text { aerofoil span } \\ C & =\text { aerofoil chord } \\ c & =\text { wavelet coefficient } \\ C & =\text { aerofoil effective chord length } \\ f_{s} & =\text { hot-wire signal sampling frequency } \\ P W & =\text { square waveform signal pulse width } \\ t_{T E} & =\text { tripping element thickness } \\ x & =\text { streamwise distance along chord } \\ \alpha & =\text { angle of attack } \\ \alpha_{g} & =\text { geometrical angle of attack } \\ \gamma & =\text { turbulence intermittency factor } \\ \theta & =\text { microphone polar angle } \\ \lambda & =\text { serration wavelength }\end{array}$

\footnotetext{
${ }^{1} \mathrm{PhD}$ Student, Department of Mechanical and Aerospace Engineering, non-member AIAA.

${ }^{2}$ Senior Lecturer, Department of Mechanical and Aerospace Engineering, member AIAA.
} 


\section{Introduction}

Despite the increasing number of large offshore wind farm projects, on land wind turbines still are the prevalent method of harnessing wind energy. A publication by Wind Europe outlines several scenarios for future development of wind energy infrastructure in Europe. According to the Central scenario, of $323 \mathrm{GW}$ of total wind power capacity installed until 2030, $253 \mathrm{GW}$ (or 78\%) are expected to come from onshore and $70 \mathrm{GW}$ (or 22\%) from offshore wind turbines ${ }^{1}$. The capacity of wind generated power is therefore expected to be more than double relative to the level recorded in 2016, indicating that power production from wind is likely to be significant in terms of power production in the future. Moreover, due to majority ( $78 \%$ ) of wind power coming from land-based wind turbines, it is anticipated that the number of communities living in close proximity to them will be increased. This can potentially impose a limit on a more widespread production of onshore wind power due to concern with the community noise exposure related issues. Evidently, there is a need for research focusing on wind turbine noise reduction technologies.

When considering the overall noise produced by wind turbines, the main contribution is normally attributed to the blade-generated aerodynamic noise. As described in ${ }^{2}$, the latter is dominated by the turbulent boundary layer - trailing edge (TBL-TE) broadband noise outlined in ${ }^{3}$. The generation of this type of noise has been addressed by a number of passive flow control methods, such as brushed trailing edges, finlets and different types of bio-inspired trailing edge serrations. Flat-plate add-on type serrations, however, have attracted the most research attention due to their simplicity and strong ability to reduce aerofoil trailing edge broadband noise ${ }^{4-10}$. Although many reports on serrated aerofoils can be found in the literature, a consensus with regard to the exact mechanism through which trailing edge serrations can achieve noise reductions has not been reached.

A study presented in ${ }^{11}$ has partly focused on the steady and unsteady aerodynamics around an aerofoil to provide more insight into the mechanism of trailing edge noise reduction by serrations. Six flat plate trailing edge serration configurations with varying serration amplitudes and wavelengths have been tested on a NACA 651210 cambered aerofoil placed in an open-jet wind tunnel at a $5^{\circ}$ angle of attack. The study has observed that the boundary layer profiles measured along the span of the root of a single serration sawtooth have shown only minor differences in comparison to the profiles measured at the corresponding locations on a straight trailing edge. This indicates that serrations only have a minor or no effect on the boundary layer upstream of serrations. Similarly, turbulent velocity profiles in the boundary layer for baseline and serrated cases tend to become more comparable as the measurement position shifts closer to the serration root, although a $30 \%$ increase in maximum turbulence intensity is observed in the serration case, even at the serration root. At the serration tip, however, mean velocity profile is $15 \%$ thicker when compared to the baseline case and for this reason turbulent velocity profiles indicate turbulence being shifted away from the surface.

Part of an experimental study in ${ }^{12}$ used a liquid crystal technique to visualise the effect of turbulent flow passing through a serrated trailing edge on a flat plate. Pre-heated serration surface allowed crystals to change colour in relation to an increase or decrease in heat transfer, which provides information about the different flow regimes. The study identified higher time-averaged heat transfer rates at the side edges and tips of serrations, implying that this was induced by a stronger flow regime than a turbulent boundary layer. This strongly correlated to the surface pressure fluctuation measurements, where higher wall pressure spectral energy levels have been located near the serration edges and tips. The authors related these observations to the presence of convective pressure-driven vortical structures that form at the serration edges (and convect into the tip region) and are a potential contributor to the broadband noise reduction. A study in ${ }^{13}$ conducted surface-pressure fluctuation measurements and showed that broadband noise reduction by serrations is due to the major reduction in phase speed of turbulence convection near saw-tooth edges as well as the reduction in coherence of pressure measurements along the serration edge.

Strong support towards the observations described in the previous paragraph is provided by a study in ${ }^{14}$. Timeresolved tomographic particle image velocimetry PIV has been used to study the three-dimensional flow field on the suction side of a serrated NACA 0018 aerofoil placed in an open-jet wind tunnel with a geometric angle of attack of $4^{\circ}$. Straight and serrated trailing edge cases have shown strong similarities in terms of mean velocity and turbulent fluctuation boundary layer profiles, indicating that the turbulent boundary layer upstream of serrations is only weakly affected by their presence and this is in agreement with ${ }^{11}$. Downstream of serrations, however, the flow becomes complex with streamwise-oriented vortical structures forming around the serration edges and additional counter-rotating flow structures that are present in the serration gaps. These structures are believed to induce secondary effects, such as funnelling of the incoming flow which in turn changes the serration effective angle $\varphi^{\prime}$. This has also been reported in ${ }^{12}$ and it is believed that this phenomenon could contribute to the way broadband noise is scattered along the oblique edges. The funneling effect is also evident from the contours of time averaged streamwise velocity component at the serration surface that show the flow approaching the serrations seeping into the serration gaps. This observation has later been verified by a numerical study in ${ }^{15}$, where simulations on a serrated NACA 0018 aerofoil under zero lift conditions have shown that the near-wall $(z$ $=0.5 \mathrm{~mm}$ from the wall) spatial distribution of time averaged mean streamwise velocity component has shown an increase when moving away from the root towards the tip, indicating an acceleration of the flow. 
Numerous analytical models exist for the prediction of aerofoil trailing edge noise. Amiet derived an expression at low Mach number flow for the radiated far field spectra as a function of the wall pressure spectrum upstream of the trailing edge and spanwise correlation length ${ }^{16}$. The hydrodynamic pressure field, which is induced by the turbulent boundary layer unsteady velocity field, represents a key parameter for the self-noise radiation. The classic Blake-TNO model ${ }^{17}$ enables the prediction of turbulent boundary layer - trailing edge broadband noise based on modeling of the wall pressure fluctuation upstream of the trailing edge. For the unsteady surface pressure wavenumber frequency spectrum $P(k, \omega)$ it can be expressed as

$$
P(k, \omega)=4 \rho^{2}\left(\frac{k_{1}^{2}}{k_{1}^{2}+k_{3}^{2}}\right) \int_{0}^{\infty}\left[\frac{d U_{1}\left(x_{2}\right)}{d x_{2}}\right]^{2} \cdot \Lambda_{2}\left(x_{2}\right) \cdot \tilde{\varphi}_{22}(k) \cdot u_{2}^{2}\left(x_{2}\right) \cdot \varphi_{m}\left(\omega-k_{1} U_{c}\right) e^{-2|k| x_{2}} d x_{2}
$$

where $\Lambda_{2}\left(x_{2}\right)$ is the vertical integral length scale, $\varphi_{m}$ is the moving axis spectrum for the assumption of frozen turbulence passing over the trailing edge, and $\tilde{\varphi}_{22}(k)$ is the vertical velocity fluctuation spectral density. The expression in Eq. 1 also identifies the contribution of the wall-normal fluctuating velocity component, $v^{\prime}\left(u_{2}\right.$ in the equation), to the far field noise radiation. This implies that reduction of $v^{\prime}$, amongst other parameters described in Eq. 1, has a potential to reduce the level of noise radiation.

The present work aims to investigate the temporal and spatial development of the flow structure in close vicinity of aerofoil trailing edge by means of flow measurements using hot-wire anemometry. This is achieved by employing a technique that allows replicating the process of natural formation of 'packets' of turbulence, known as turbulent spots, seen in the later stage of boundary layer transition. It is normally observed that at this stage, individual turbulent spots begin to appear randomly (in both space and time) and intermittently. After their initial appearance, turbulent spots exhibit transverse, spanwise and streamwise growth as they are carried along by the freestream flow. The streamwise growth is mainly governed by the difference in propagation rates at the leading and trailing edges of the turbulent spot, causing the spot to stretch as it propagates downstream ${ }^{18}$. Growth in the transverse direction occurs when turbulent eddies within the turbulent spot entrain the high momentum irrotational fluid from the freestream into the turbulent spot ${ }^{19}$. The lateral, or spanwise, growth is due to destabilisation of the fluid surrounding the spot. The principal mechanism explains that the turbulent eddies within the spot induce perturbations into the surrounding unstable laminar boundary layer, which then grow rapidly and eventually break down, inducing new turbulence ${ }^{20}$. Due to this spanwise growth, turbulent spots are characterised by the spreading half-angle of $9-10^{\circ}$ under the conditions of zero streamwise pressure gradient ${ }^{21}$. Due to this growth, the individual turbulent spots, sometimes referred to as the 'building blocks' of the turbulent boundary layer, begin to interact with each other at a sufficient distance downstream. The extent of transitional boundary layer can be described by the turbulence intermittency factor $\gamma$, or the fraction of time for which the boundary layer is turbulent ${ }^{22}$, which covers the range of $0<\gamma<1$. Through the process of interaction, individual spots exchange momentum and coalesce into a fully turbulent boundary layer, reaching the intermittency factor of $\gamma=1$.

The experimental technique proposed here relies on generating a momentarily induced synthetic turbulent boundary layer, as a result of coalescence of a number of artificially generated turbulent spots, in a controlled manner. The applicability of the proposed technique for the study of the interaction between turbulent boundary layer and trailing edge serrations can be validated by findings from several different studies. First, strong similarities between naturally occurring and artificially generated turbulent spots are described in ${ }^{18}$. In addition, research studies described in ${ }^{23,24}$ have identified similarities between the turbulent spots and the turbulent boundary layer in terms of their structure and mechanisms of growth. Further validation to the proposed experimental technique is provided in ${ }^{25}$. The study compares some properties (such as mean velocity, turbulence intensity, ensemble phase-averaged velocity) of a synthetically generated turbulent boundary layer and that induced by a two-dimensional trip (also used as reference flow). It was generally observed that synthetic turbulent boundary layers (hereafter referred to as SBL) and tripped or naturally turbulent boundary layers show similar development of the mean flow at sufficient distances from the generation location and any differences in mean flow parameters is caused by shifts in the origins of boundary layer development. The strong similarity between a turbulent boundary layer and a SBL opens up a possibility to employ a technique that enables one to demonstrate the temporal-spatial evolution of a turbulent boundary layer as it passes through the measurement volume or measurement plane of interest. This provides more information on the flow than the more common approach where the mean or fluctuating properties of a fully established turbulent flow are studied. A detailed discussion on the methodology is presented in Section III.B. 


\section{Experimental setup and analysis techniques}

\section{A. Wind tunnel facilities and instrumentation}

Aerodynamic noise measurement and flow measurement experiments were performed at Brunel University London in the low-speed wind tunnel, described in more detail in ${ }^{26}$. This open-loop open jet aero-acoustic wind tunnel has a test section of $0.1 \times 0.3 \mathrm{~m}^{2}$ and produces a freestream flow with $0.1-0.2 \%$ turbulence intensity.

Far field measurements of trailing edge broadband noise are performed using a polar array of eight $1 / 2$ inch G.R.A.S. 46AE condenser microphones connected to two G.R.A.S. 12AX CCP power modules. The array is located at $0.97 \mathrm{~m}$ from the mid-span of the aerofoil trailing edge and the microphones are placed at polar angles of $\Theta=50-120^{\circ}$ with an interval of every $10^{\circ}$. The aerofoil is placed in the freestream jet at a $0^{\circ}$ geometrical angle of attack $\alpha_{g}$. Providing that the aerofoil is symmetrical and no angle of attack is applied, the effective angle of attack is also assumed to be $\alpha_{\mathrm{eff}}=0^{\circ}$. Boundary layer transition is induced by introducing a zig-zag shaped tripping element (having a thickness of $t=0.6 \mathrm{~mm}$ ) at $x / C=0.2$ from the leading edge on both aerofoil surfaces.

The current study investigates the temporal development of the SBL that was mentioned in the introduction. In order to supplement this data by the temporal variation of the noise emitted by the aerofoil trailing edge, it was necessary to collect SBL noise measurement data that are subsequently used for wavelet analysis (this will be described in more detail in Section C.4). Data are sampled by two G.R.A.S. 46AE condenser microphones that are placed each side of the mid-span of the aerofoil trailing edge at polar angles of $\pm 90^{\circ}$ and a distance of $0.21 \mathrm{~m}$. This experiment used an acoustic test section with Kevlar (plain weave Kevlar $120_{\circledast}$ cloth, 58 g/m²) walls, design of which is mostly based on findings in ${ }^{27}$. Tensioned panels of Kevlar cloth are installed such that they cover the upper and lower sides of the jet exiting the nozzle, permitting the sound to pass and be measured while containing the bulk of the flow. Note that the sound pressure readings are not corrected for losses due to the transmission of sound through the Kevlar walls and the boundary layer that is formed on the Kevlar wall. As a result, the absolute level of the measured sound pressure is lower than the actual pressure emitted by the aerofoil trailing edge.

The wind tunnel is equipped with a three-dimensional traverse system, having a resolution of $\pm 0.01 \mathrm{~mm}$ in all three axes, to facilitate flow measurements using hot-wire anemometry. Similarly to the aerodynamic noise measurements, the aerofoil is mounted in the test section at a $0^{\circ}$ geometrical angle of attack $\alpha_{\mathrm{g}}$. Both the aeroacoustic and aerodynamic measurements were conducted under the same flow conditions and using a test section fitted with Kevlar walls.

\section{B. Aerofoil design and artificial turbulent spot generation}

The aerofoil being investigated is a NACA 0008 symmetrical profile with a $0.15 \mathrm{~m}$ chord length $C$ and a 0.3 $\mathrm{m}$ span $b$ as shown in Fig. 1. The main body of the aerofoil comprises two halves that make up the full NACA 0008 profile. A profile with low thickness to chord ratio $t / c$ has been selected to reduce the level of adverse pressure gradient experienced by the aerofoil surfaces at the aft region. This is expected to prevent early boundary layer separation that would render the proposed experimental technique unfit for use at the Reynolds number of interest. The reason for this becomes clearer in Section C.2.

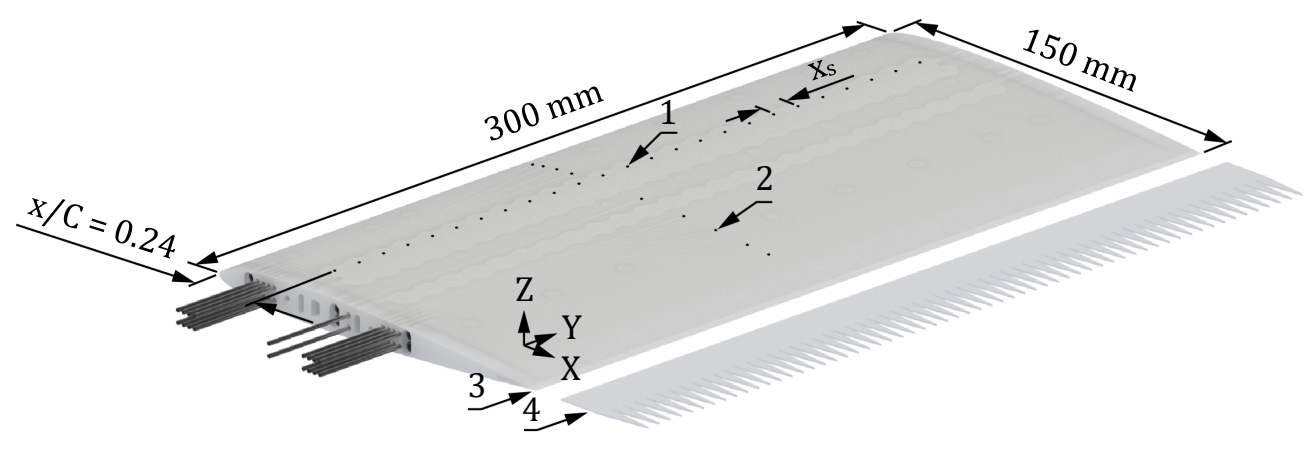

Fig. 1 NACA 0008 aerofoil and its main features: $1-0.5 \mathrm{~mm}$ diameter orifice; $2-0.5 \mathrm{~mm}$ diameter static pressure tap; 3 - Straight (baseline) trailing edge; 5 - Serrated $(\lambda 5 h 15)$ trailing edge; $x_{s}$ - spacing between individual orifices

The aerofoil trailing edge is designed to have a slit that accommodates two types of flat plate insert: 1) Straight insert that acts as a baseline case; 2) Serrated insert. In the current study, trailing edge is fitted with saw-tooth type serrations having a $5 \mathrm{~mm}$ wavelength, $\lambda$, and a $15 \mathrm{~mm}$ amplitude, $2 h$, corresponding to a wavelength to amplitude ratio of $\lambda / h=0.33$. Serrations are applied to the aerofoil such that they extend from the straight (baseline) trailing edge, hence increasing the effective chord length of the aerofoil by the length of the serration amplitude $(2 h)$ as shown in Fig. 2. The serration root therefore coincides with the trailing edge of the baseline aerofoil. 
The experimental technique used in the current study relies on the method of artificial generation of a SBL that varies both in time and space. A similar methodology has previously been adapted for flow measurements on a flat plate in ${ }^{25}$. SBL is formed by inducing a number of closely positioned individual turbulent spots, each of them being generated by introducing a small disturbance into an otherwise laminar flow on the aerofoil surface. The disturbance is induced by generating a jet through the displacement of the diaphragm of each of the miniature speakers (see Fig. 3 ), which in turn forces the air out of an orifice in the aerofoil surface. Providing that the magnitude of the jet is sufficiently large and additional momentum is transferred into the boundary layer, a turbulent spot can be induced at some distance downstream. Such turbulent spot generation

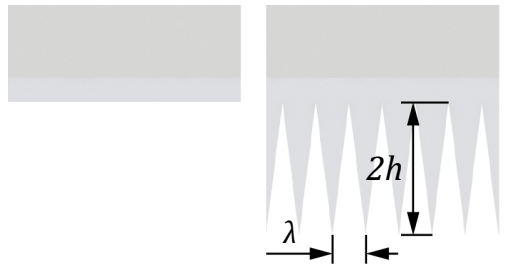

Fig. 2 Baseline, left, and serrated ( 25 h15), right, trailing edges; $2 h$ serration amplitude, $\lambda$ - serration wavelength method has been employed previously in ${ }^{21,28,29}$ and others. The jets are discharged from a transverse array (present on both aerofoil sides) of twenty-five $0.5 \mathrm{~mm}$ diameter orifices spaced $x_{s}=11 \mathrm{~mm}$ apart, all of which are located at $x / C=0.24$, refer to Fig. 1 . This generates an array of individual turbulent spots that produce a momentarily induced array of turbulence that travels downstream of its origin along with the freestream flow. As a result of the principal mechanisms outlined in the introduction, the turbulent spots gradually grow in streamwise, spanwise and wall normal directions as they propagate downstream. At some distance downstream of their origin, the individual turbulent spots begin to interact and eventually coalesce to form a uniform turbulent flow, which was referred to as the synthetic turbulent boundary layer (SBL). It is important to note that the actuation of the upper and lower arrays of the miniature loudspeaker are synchronised. Because the aerofoil is set at an angle of attack of $0^{\circ}$, the SBL on both upper and lower sides will arrive at the trailing edge at the exact same instance.

The speakers are driven by a $12 \mathrm{~Hz}$ square pulse waveform signal (having a pulse width $P W$ of 1.7 milliseconds). The rising edge of the pulse is used as a reference for ensemble phase-averaging of the flow measurement data. The chosen spot generation settings have proven to generate turbulent spots comparable to those seen in other studies ${ }^{30}$.

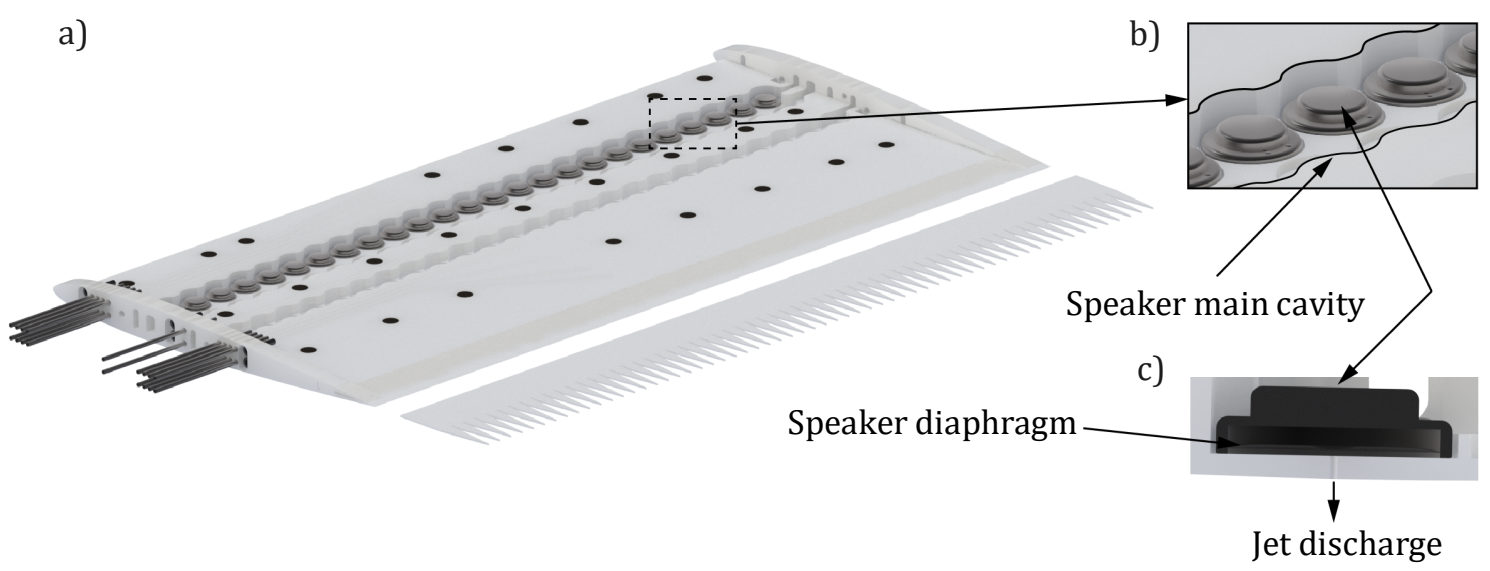

Fig. 3 Aerofoil internal features: a) Main body - bottom half; b) Close-up view of speaker main cavity and miniature speakers; c) Cross-sectional view of an individual miniature speaker

The generated SBL, along with the freestream flow, convects downstream from its origin and eventually passes through the trailing edge and the point where the measurements are taken. Providing that the signal of the velocity fluctuations induced by the passage of the SBL can be distinguished from the signal of an otherwise undisturbed flow, signature of velocity fluctuations caused by each passage of SBL are measured using hot-wire anemometry. Data are sampled to record a large number of individual events of SBL passage at each measurement point. They are then analysed using the method outlined in the following section to reconstruct the temporalspatial development of the structure of the SBL as it passes through the straight, or serrated, trailing edge.

\section{Data collection and processing methods}

\section{Collection of hot-wire anemometry data}

Experiments are conducted by collecting velocity fluctuation data at $U_{\infty}=9.5 \mathrm{~ms}^{-1}$, corresponding to a chordbased Reynolds number of $0.96 \times 10^{5}$. All measurements are taken using a DANTEC 55P61 miniature X-wire probe, where both wires are operated at an overheat ratio $a$ of 1.8 with a constant-temperature anemometer. The signal is sampled at $5 \mathrm{kHz}$ for $26.2 \mathrm{~s}$ after passing through a low-pass anti-aliasing filter and is digitised by a 12- 
bit analog-to-digital data acquisition card. Data set of a single measurement point contains 131,072 samples and velocity signatures of 341 individual turbulent spots.

Measurements are taken across selected two-dimensional X-Y and Y-Z planes, set up with reference to the coordinate system indicated in Fig. 4 or Fig. 5. All measurement planes are positioned using a common reference point, located at $8 \mathrm{~mm}$ upstream of the baseline trailing edge as well as the corresponding location on a serrated trailing edge.

Data of the streamwise velocity component $u$ (positive along the $\mathrm{X}$ axis) and the transverse, or wall-normal, velocity component $v$ (positive along the $\mathrm{Z}$ axis) are collected in the $\mathrm{X}-\mathrm{Y}$ measurement planes that cover an area of $6 \times 9 \mathrm{~mm}^{2}$ and $6 \times 24 \mathrm{~mm}^{2}$ for the baseline and serrated trailing edge cases respectively. The planes are illustrated in Fig. 4. Measurements are taken across planes located at $z_{p}=0.6,1.3,1.7,2.5$ and $3 \mathrm{~mm}$. Dimensions of the planes are chosen such that there is an overlap of $0.5 \mathrm{~mm}$ on the each side of the serration along the $\mathrm{Y}$ axis.

(a)
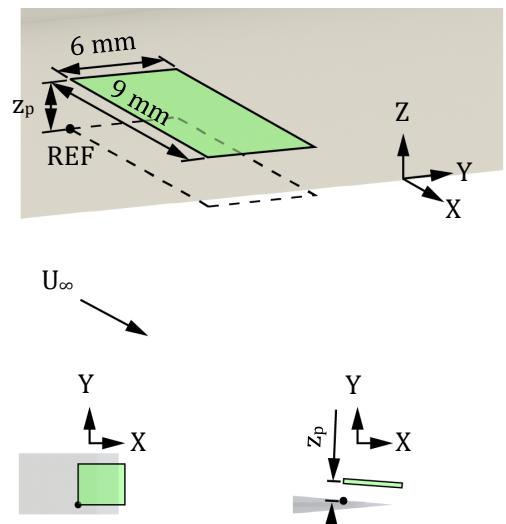

Top view

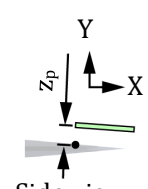

Side view

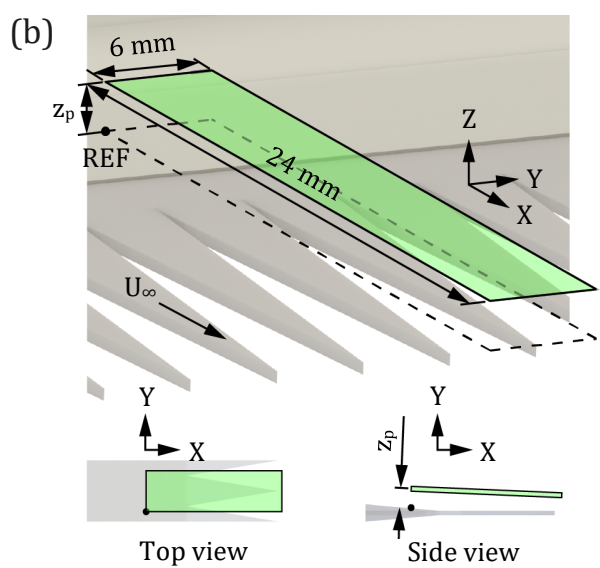

Fig. 4 Illustration of the X-Y measurement planes on (a) baseline, and (b) serrated aerofoil trailing edges; 'REF' indicates the location of the reference point

Data of the streamwise $u$, the transverse $v$ and the spanwise $w$ (positive along the $\mathrm{Y}$ axis) velocity components are collected in the Y-Z planes that cover an area of $6 \times 13 \mathrm{~mm}^{2}$ for both baseline and serrated trailing edge cases. Measurements are taken across the planes that are located in the near wake $(0.5 \mathrm{~mm}$ from the tip) of the baseline and serrated aerofoils and are positioned such that the mid-point (along $\mathrm{Z}$ axis) of the plane coincides with the tip of both baseline and serrated aerofoil trailing edge as demonstrated in Fig. 5.

(a)
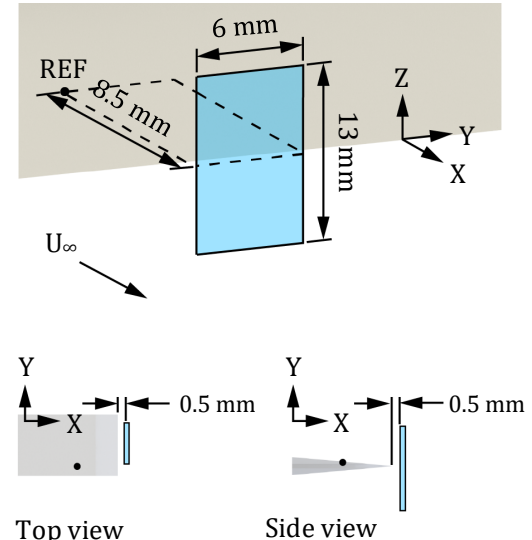

(b)

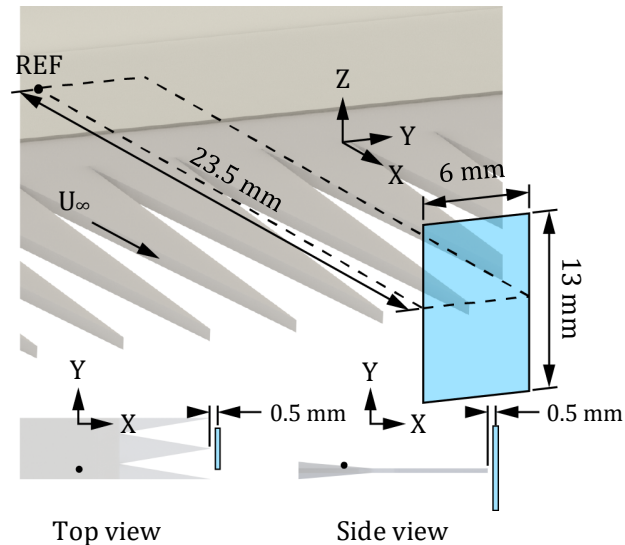

Fig. 5 Illustration of the Y-Z measurement planes on (a) baseline, and (b) serrated aerofoil trailing edges

\section{Analysis of hot-wire anemometry data}

As explained in section B, the SBL is formed by a number of individual turbulent spots. The current study therefore employs data analysis methods that are commonly used in the investigation of the characteristics of isolated (individual) turbulent spots. The following will describe those methods in greater detail.

Data set of a single measurement point contains 131,072 samples and velocity signatures of 341 individual SBL passage events. The rising edge of the square pulse (used to drive the miniature speakers), which represents the time of origin, $t=0$, locates the signal of each individual generation. This definition is used to generate an 
ensemble that is averaged to obtain the mean velocity signature generated by the SBL at the point of measurement as illustrated by Fig. 6a. Typical mean velocity signatures measured across one local boundary layer thickness are shown in Fig. 6 b.

(a)

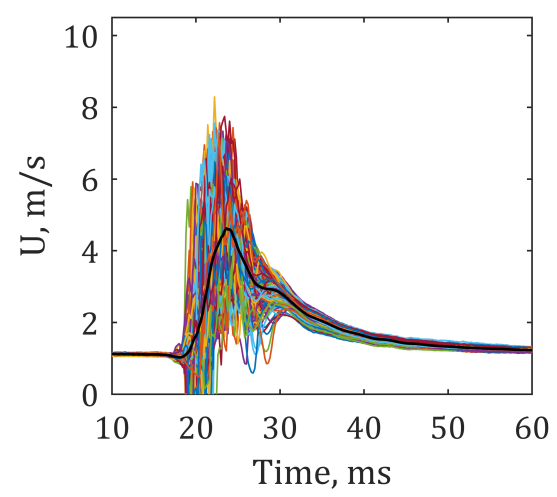

(b)

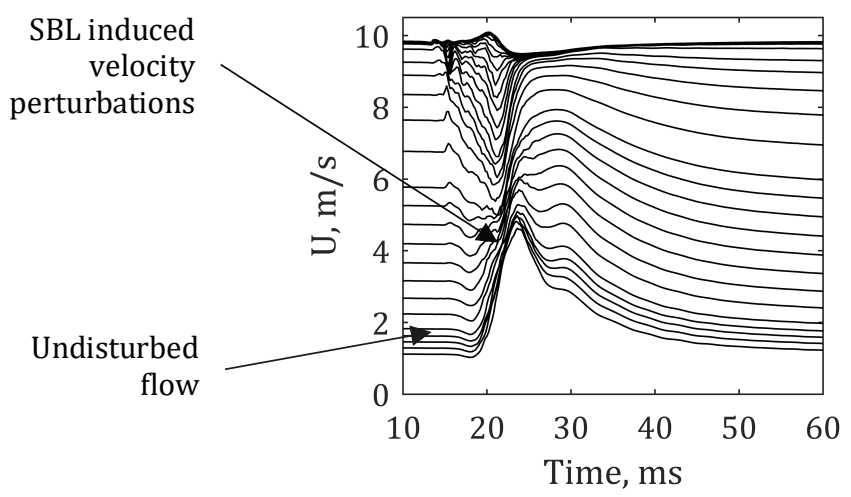

Fig. 6 (a) An ensemble of 341 turbulent spot velocity signals (coloured lines) overlapped with an ensemble phase-averaged velocity signature (black solid line), measured at $z_{p}=0.1 \mathrm{~mm}$ from the trailing edge surface, and (b) ensemble phase-averaged velocity signatures measured at different $z$ locations from the surface

Velocity perturbation, which measures the momentum excess or deficit caused by the SBL, are obtained by scaling the SBL mean velocity with the local freestream velocity $U_{\infty}$. If temporal variation of the ensembleaveraged streamwise, transverse and spanwise velocity components are denoted by $\langle U(x, y, z, t)\rangle,\langle V(x, y, z, t)\rangle$ and $\langle W(x, y, z, t)\rangle$ respectively, then the velocity perturbation, $\widetilde{U}, \widetilde{V}$ and $\widetilde{W}$ can be expressed by:

$$
\begin{aligned}
\widetilde{U}(x, y, z, t) & =\frac{\langle U(x, y, z, t)\rangle-U_{l}(x, y, z)}{U_{\infty}(x)} \\
\widetilde{V}(x, y, z, t) & =\frac{\langle V(x, y, z, t)\rangle-V_{l}(x, y, z)}{U_{\infty}(x)} \\
\widetilde{W}(x, y, z, t) & =\frac{\langle W(x, y, z, t)\rangle-W_{l}(x, y, z)}{U_{\infty}(x)}
\end{aligned}
$$

where $t$ is time, $U_{l}, V_{l}$ and $W_{l}$ are the local velocities of the undisturbed flow and $U_{\infty}$ is the velocity of the freestream flow.

Similarly, the temporal variation of ensemble-averaged rms velocity fluctuations, $u^{\prime}, v^{\prime}$ and $w^{\prime}$, can be determined by:

$$
\begin{gathered}
u^{\prime}(x, y, z, t)=\sqrt{\frac{\sum_{i=1}^{N}\left[U(x, y, z, t)-\langle U(x, y, z, t)]^{2}\right.}{N}} \\
v^{\prime}(x, y, z, t)=\sqrt{\frac{\sum_{i=1}^{N}\left[V(x, y, z, t)-\langle V(x, y, z, t)]^{2}\right.}{N}} \\
w^{\prime}(x, y, z, t)=\sqrt{\frac{\sum_{i=1}^{N}[W(x, y, z, t)-\langle W(x, y, z, t)\rangle]^{2}}{N}}
\end{gathered}
$$


where $N$ is the number of realisations. Finally, the temporal variations of the Reynolds shear stress $\left\langle u^{\prime} v^{\prime}\right\rangle$ can be calculated from the following equation:

$$
\left\langle u^{\prime} v^{\prime}\right\rangle(x, y, z, t)=\frac{\sum_{i=1}^{N}[U(x, y, z, \tau)-\langle U(x, y, z, \tau)\rangle][V(x, y, z, \tau)-\langle V(x, y, z, \tau)\rangle]}{N}
$$

The reduced data allows each measurement point to be assembled to generate contour plots of velocity perturbation in time domain, such as the example in Fig. 7a. The contour plot represents changes in flow momentum induced by the SBL relative to local undisturbed flow, where positive or negative values denote momentum increase or decrease respectively. Similarly, velocity fluctuation contour is interpreted as change in turbulence intensity of the flow, which is illustrated in Fig. 7b.

(a)

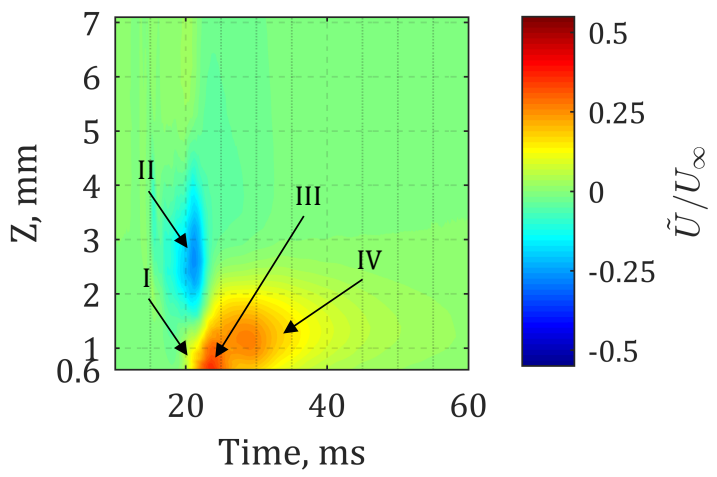

(b)

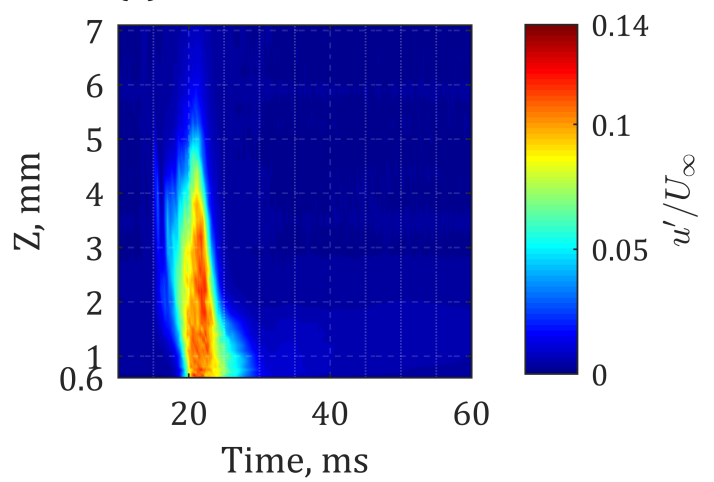

Fig. 7 (a) Velocity perturbation $\widetilde{U} / U_{\infty}$, and (b) rms velocity fluctuation $u^{\prime} / U_{\infty}$ contour plots

Closer investigation of the velocity perturbation contour (Fig. 7a) demonstrates a structure that is typical to turbulent spots, providing an indication that the SBL is in fact a formation of turbulent spots as initially anticipated. The general structure of a turbulent spot is first described by the leading edge overhang that is located in Region I (see Fig. 7a). Directly behind is the positive perturbation region (III) that carries a large fraction of the momentum and above it is the negative perturbation region (II). The so-called calm region is indicated by Region IV and literature suggests that this region possesses high stability relative to the surrounding fluid, significantly more stable than the surrounding laminar flow, implying that destabilisation of this region can only occur upon interaction with a strong flow regime.

3. Far field measurements of fully developed TBL - TE broadband noise (stationary signals)

Noise data were sampled for $20 \mathrm{~s}$ at $40 \mathrm{kHz}$ sampling frequency by a 16-bit analog to digital card from National Instruments the mean flow velocities ranging $20-40 \mathrm{~ms}^{-1}$. The data is then windowed and the Power Spectral Density (PSD) of $1 \mathrm{~Hz}$ bandwidth is computed from a 1024 point FFT. Although acoustic data was collected using 8 microphones, measurement results of a microphone placed at a polar angle of $\Theta=80^{\circ}$ to the aerofoil trailing edge are presented.

4. Measurements of SBL - trailing edge noise (non-stationary signals) for wavelet analysis

A wavelet analysis is used to investigate the temporal variations in the energy content and frequency of a signal that measures far field acoustic pressure fluctuations caused by the passage of the SBL through an aerofoil trailing edge. The signal is sampled for $26.2 \mathrm{~s}$ at $40 \mathrm{kHz}$ sampling frequency by a 12-bit A/D card using the microphone setup described in Section A. The analysis uses a continuous wavelet transform (CWT) and employs the Morlet wavelet, choice of which is based on the similarity to a typical signal of acoustic pressure fluctuations. Similarly to the method used in the processing of the velocity signal measured using hot-wire anemometry, the wavelet coefficient data obtained from CWT is ensemble-averaged to obtain the mean wavelet coefficient data. The latter are used to identify short time variations in the magnitude of acoustic pressure waves scattered into the far-field due to the hydrodynamic interaction between the straight or serrated aerofoil trailing edge and the SBL that passes through it. 


\section{Results}

\section{Noise measurement data}

\section{Stationary turbulent boundary layer - trailing edge broadband noise}

Aero-acoustic measurement experiments were conducted to establish that the configuration of trailing edge serrations selected for the current study can reduce the far field noise radiated by the aerofoil trailing edge. Any reduction in the observed level of noise radiation would imply that the serrations modify the noise scattering characteristics of the aerofoil trailing edge and therefore must create changes in the flow field in the near vicinity of trailing edge serrations. Results are demonstrated in Fig. 8. The level of noise reductions achieved by the serrations is illustrated by a contour map of $\triangle S P L$ that is expressed as $S P L_{\text {baseline }}-S P L_{\text {serrated. }}$. A positive level of $\triangle S P L$ therefore represents a noise reduction and vice versa. The contour shows that noise reductions can be achieved across the entire range of Reynolds numbers and the frequencies in the range of $1.3-9 \mathrm{kHz}$. As anticipated, the frequency spectra shown in Fig. 8a indicate that at the freestream flow velocities of $U_{\infty}=30$ and $40 \mathrm{~ms}^{1}$ the noise radiation is broadband in nature.

(a)

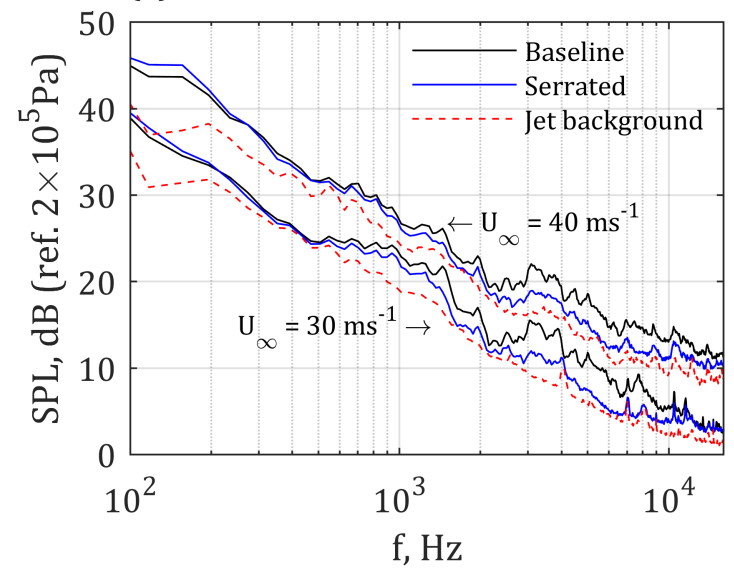

(b)

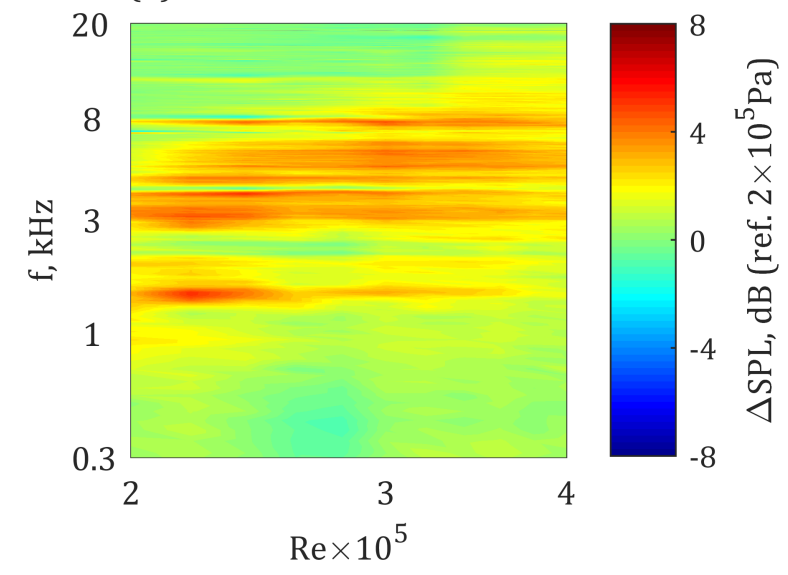

Fig. 8 (a) Spectra of SPL measured at $U_{\infty}=30$ and $40 \mathrm{~ms}^{-1}$, and (b) contour map of $\triangle S P L$ produced by $\lambda 5 \mathrm{~h} 15$ saw-tooth serrated trailing edge. Microphone is placed at a polar angle of $\Theta=80^{\circ}$

2. Time varying synthetic turbulent boundary layer - trailing edge noise

The wavelet coefficients obtained from the continuous wavelet transform (CWT) of acoustic pressure measurements are demonstrated in the CWT scalogram in Fig. 9. The contour depicts values of scaled wavelet coefficients, denoted as $c_{w}=10 \cdot \log _{10}(c)$, where $c$ is the wavelet coefficient expressed in terms of acoustic pressure. Measurements were taken at $\mathrm{Re}=0.96 \times 10^{5}$ using a microphone placed over the top surface of the aerofoil. Results obtained from the measurements of the microphone facing the bottom surface (i.e. $\Theta=270^{\circ}$ ) have demonstrated similar results and are therefore not presented in this paper.

The values of $c_{w}$ indicate the temporal variation in acoustic pressure spectra emitted at the straight or serrated aerofoil trailing edge, as a result of acoustic scattering of hydrodynamic pressure waves caused by the interaction between the SBL and the straight or serrated trailing edge. Every time instance in the Fig. 9 contour maps can therefore be complemented by the flow measurement data at the corresponding time instance.

The result obtained from the baseline case measurements (Fig. 9a) identifies two pronounced regions, namely region I and region II, of energy variation in the frequency range of $120-500 \mathrm{~Hz}$. Although region I demonstrates a larger overall extent, region II contains comparatively higher amplitude variations. The serrated aerofoil case exhibits a similar pattern identifying the two distinct regions. A lack of distinct changes in Region I indicate that the flow structure responsible for this particular noise radiation is altered by the serrated trailing edge in a manner that is physically insignificant. The exact source of this noise is currently under investigation. Conversely, region II undergoes a prominent change identified by a reduction in amplitude, indicating a momentary noise reduction. This implies that, as expected, turbulence interaction with the aerofoil trailing edge is altered upon the application of trailing edge serrations. The occurrence of this is therefore associated with change in characteristics of the SBL. This will be detailed in the following sections by relating the radiated acoustic pressure at the three time instances $t_{1}=18 \mathrm{~ms}, t_{2}=22.8 \mathrm{~ms}$, and $t_{3}=25.6 \mathrm{~ms}$ (indicated by the vertical lines in Fig. 9), and the flow measurement results at the corresponding time instances. 
(a)

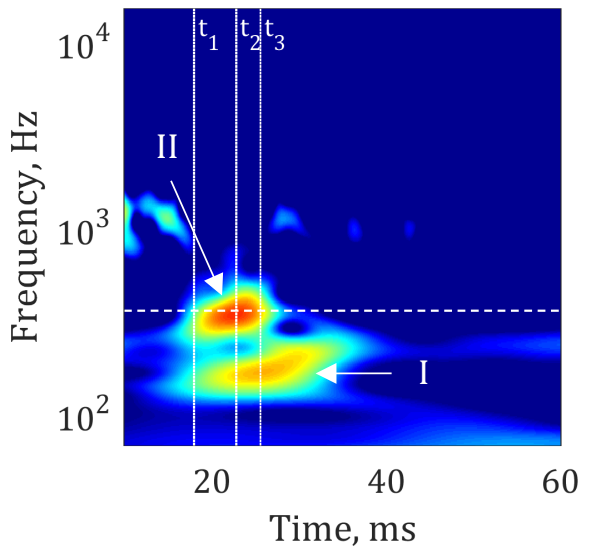

(b)

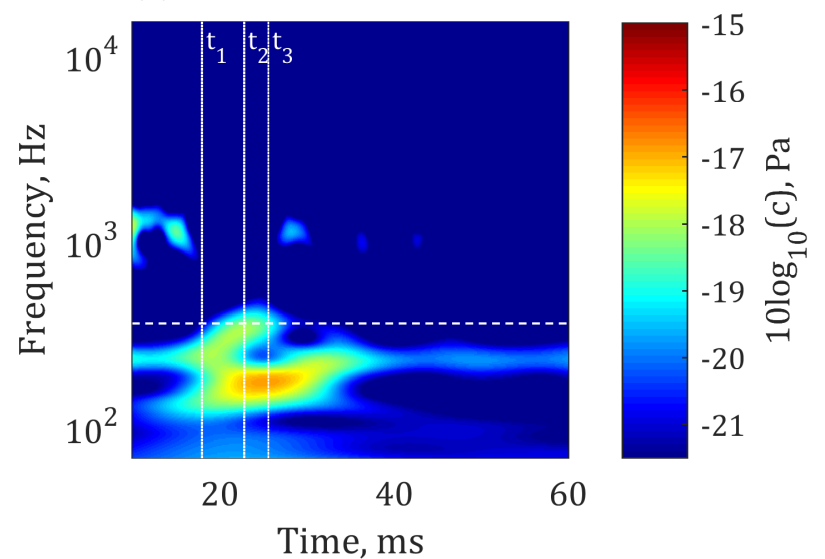

Fig. 9 CWT scalogram depicting temporal variation of the real part of scaled wavelet coefficients $c_{w}$ for the instantaneous acoustic pressure $P$ measured with the microphone placed over the top aerofoil surface. (a) baseline, and (b) serrated aerofoil, $R e=0.96 \times 10^{5}$

Here, $t_{2}$ is designated as the time instance when the peak noise radiation in Region II of straight trailing edge occurs. The precise value of $t_{2}$ is identified by plotting the time variation of the amplitude of the scaled wavelet coefficients at the frequency of $340 \mathrm{~Hz}$. The result is presented in Fig. 10. The instances when noise radiation reach their peaks are nearly identical and correspond to $t_{\max , b s}=22.8 \mathrm{~ms}$ and $t_{\max , \mathrm{s}}=22.875 \mathrm{~ms}$ for the baseline and serrated aerofoils respectively. Considering that the displacement between the two instances is only $0.075 \mathrm{~ms}, t_{2}$ $=22.8 \mathrm{~ms}$ is selected for the comparative analysis of the flow results, which will be presented in section E.2.

(a)

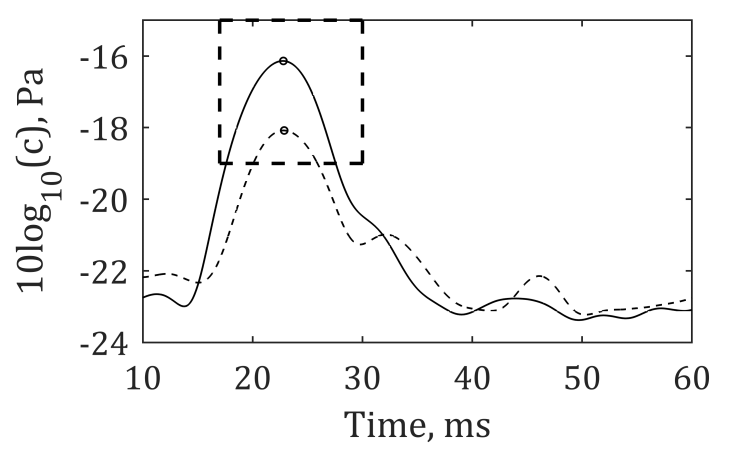

(b)

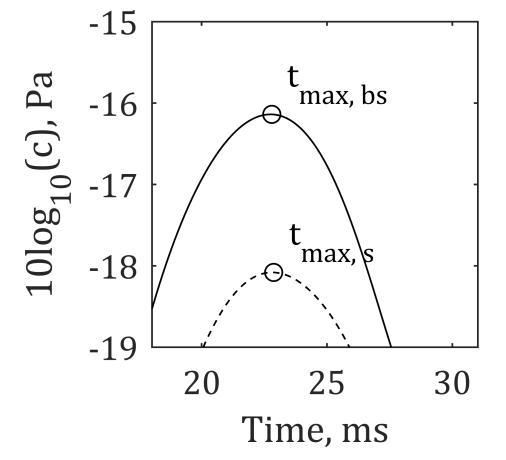

Fig. 10 (a) Scaled wavelet coefficients $c_{w}$ at $f=340 \mathrm{~Hz}$ over the full time domain, and (b) peaks isolated by the domain indicated by the dashed lines in (a)

\section{E. Flow measurement results}

\section{Turbulent spot}

Before conducting a detailed analysis of the flow measurement data, it is necessary to first establish that the artificially induced SBL is an accurate representation of a fully formed turbulent boundary layer. As shown in Fig. 11, mean velocity profile plotted across this region, or at the time instance corresponding to $t=28.8 \mathrm{~ms}$, demonstrates a profile that is typical of a turbulent boundary layer, and has a shape factor of $H=1.9$. This provides strong indication that the characteristics of the SBL resemble those of a turbulent boundary layer. A relatively high value of $H$ is expected due to the effect of an adverse pressure gradient on the boundary layer. Mean velocity profile in the laminar flow that is present before the arrival of the SBL, or at $t=11 \mathrm{~ms}$, has a shape factor of $H=$ 3 and contains comparatively less momentum near the wall as expected. 
(a)

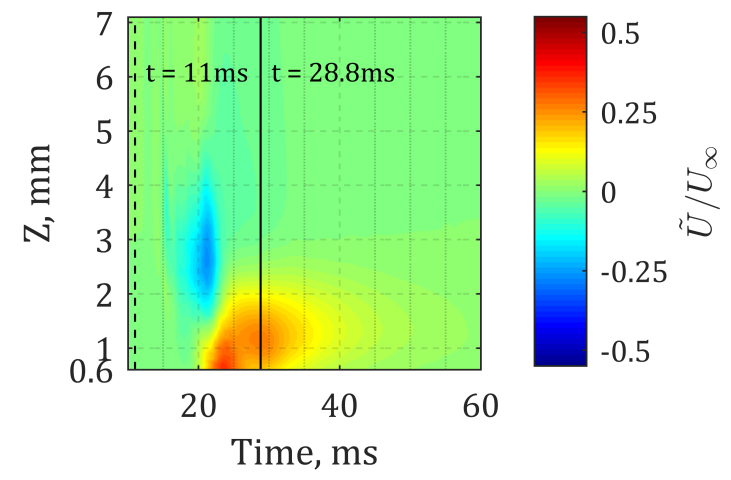

(b)

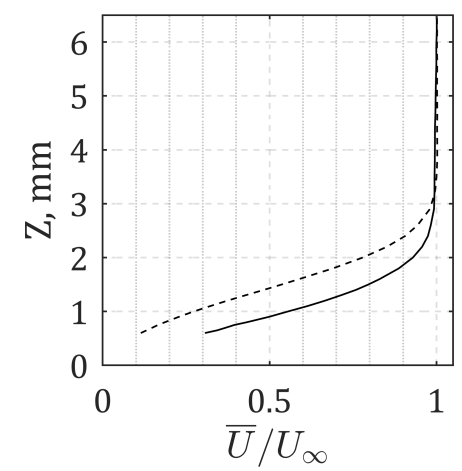

Fig. 11 (a) Contour plot depicting velocity perturbations $\widetilde{U} / U_{\infty}$ induced by the SBL, and (b) mean velocity profiles in the undisturbed flow $(t=11 \mathrm{~ms})$, dashed line, and in the turbulent region of the SBL $(t=28.8$ ms), solid line, (Right)

After the SBL is shown to be similar to a naturally occurring (or artificially tripped) turbulent boundary layer, the next step is to ensure that its development upstream of straight and serrated trailing edges is also similar.

The contour plots presented in Fig. 12 are obtained from hot-wire measurements of velocity perturbations $\widetilde{U} / U_{\infty}$ in a Y-Z plane located at $1 \mathrm{~mm}$ upstream of the serration root and the corresponding plane on a baseline trailing edge. The state of SBL is presented in three stages of the turbulent region development: arrival at $t_{1}=22.4$ $\mathrm{ms}$, fully developed region at $t_{2}=28.8 \mathrm{~ms}$ and its departure at $t_{3}=35.8 \mathrm{~ms}$. General comparison of the structures show comparable characteristics and magnitude at all three stages, suggesting similar development of SBL in both trailing edge cases. The flow upstream of serrations is therefore unaffected by their presence, as previously suggested in ${ }^{11}$. This implies that any alterations to the characteristics or the structure of the SBL at any measurement location that coincides with serrations at downstream must be caused by the presence of serrations.

(a)

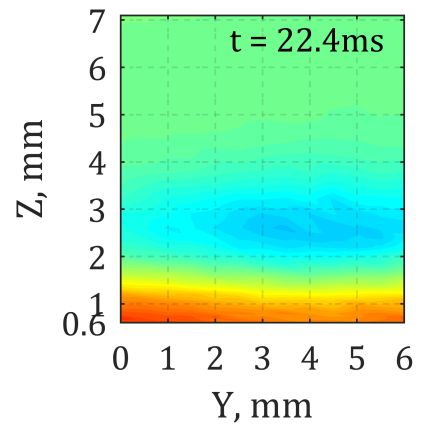

(d)

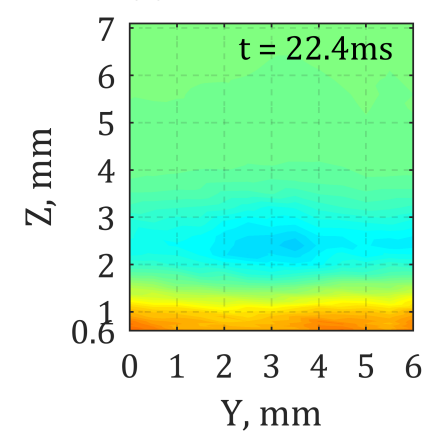

(b)

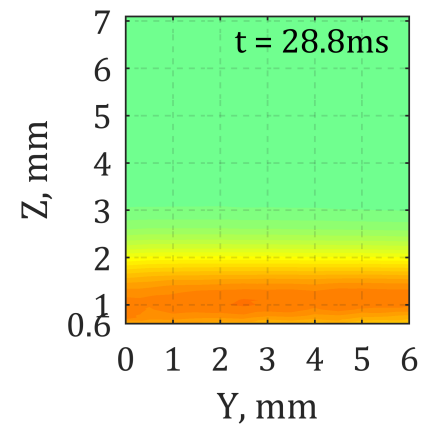

(e)

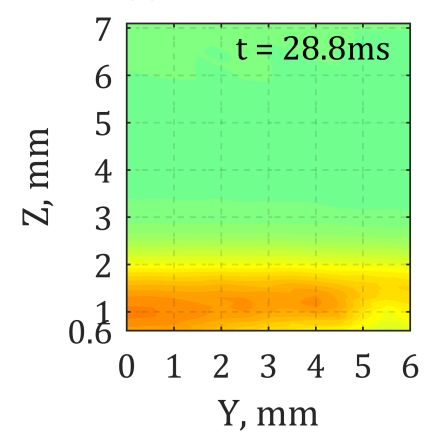

(c)

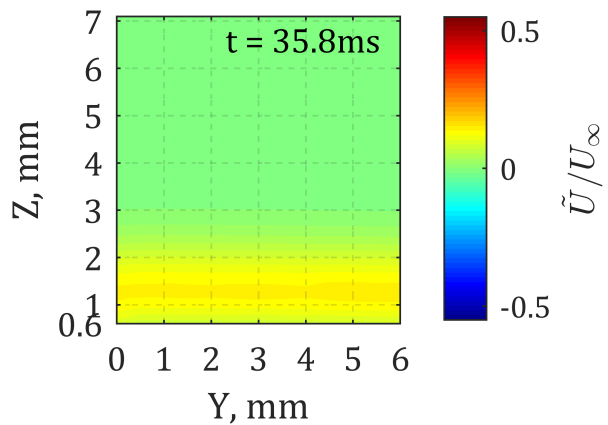

(f)

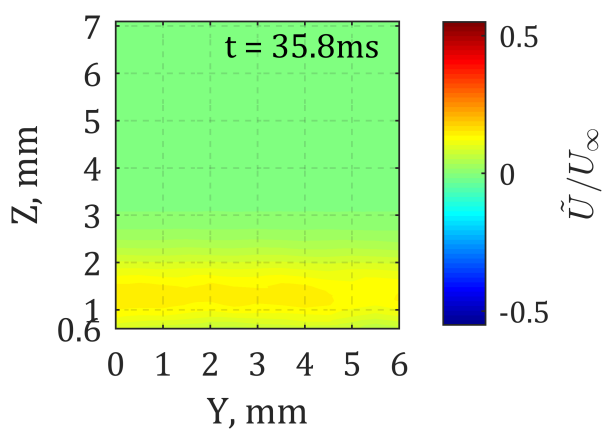

Fig. 12 Contour maps of SBL induced velocity perturbations $\widetilde{U} / U_{\infty}$ in $\mathrm{Y}-\mathrm{Z}$ plane. (a,b,c) baseline aerofoil and $(d, e, f)$ serrated aerofoil in; contours presented at $t=22.4,25.8$ and $35.8 \mathrm{~ms}$

2. Development of SBL in $X$-Y plane

A connection between the temporal variation in noise radiation and the corresponding temporal variation in flow characteristics at the three instances $t_{1}, t_{2}$, and $t_{3}$, against a backdrop of spatial domain, is discussed here. Fig. 
13 presents contour maps depicting the rms fluctuations $v^{\prime} / U_{\infty}$ in the transverse velocity component measured in the X-Y planes (refer to Fig. 4), located at $z_{p}=0.6 \mathrm{~mm}$ and $z_{p}=1.1 \mathrm{~mm}$ from the surface, at those time instances.

Considering a time instance at $t_{1}$, the contours of $c_{w}$ in Fig. $9 \mathrm{~b}$ did not indicate any occurrence of significant noise radiation. This correlates well with the contours of fluctuation in the transverse velocity component (Fig. 13a and Fig. 13b) where one of the first instances of the turbulent part of the SBL entering the measurement plane is indicated. Considering that only a fraction of the bulk turbulence contained by the SBL has reached the edge of either straight or serrated trailing edge, potential for noise scattering is therefore minimal. Results of more significance are indicated at the time instance $t_{2}$, where the peak noise reduction occurs as previously indicated. Examination of the contour suggests a general reduction in $v^{\prime} / U_{\infty}$, especially over the serrated edges. The overall effect of serrations on $v^{\prime} / U_{\infty}$ (and $\left.u^{\prime} / U_{\infty}\right)$ is quantitatively described by $u^{\prime \prime}$ and $v^{\prime \prime}$ that are estimated from double integration of $u^{\prime}$ and $v^{\prime}$ across the flow domains indicated by the dashed lines in Fig. 13c and Fig. 13d. The size of the domain is selected to include the straight edge and the serration oblique edges that are likely to be responsible for the most significant noise scattering for the baseline and serrated trailing edges respectively. $u^{\prime \prime}$ and $v^{\prime \prime}$ can therefore be obtained from the following expressions:

$$
\begin{aligned}
v^{\prime \prime} & =\frac{\iint\left(v^{\prime} / U_{\infty}\right)(x, y) d x d y}{x y} \\
u^{\prime \prime} & =\frac{\iint\left(u^{\prime} / U_{\infty}\right)(x, y) d x d y}{x y}
\end{aligned}
$$

where $x$ and $y$ are the lengths of the integration domain along the $\mathrm{X}$ and the $\mathrm{Y}$ axes respectively. Note that the size of the domains are significantly different, hence the values of integrated $v^{\prime} / U_{\infty}$ and $u^{\prime} / U_{\infty}$ are normalised by the domain size. The results are given in Table 1.

(a)

\begin{tabular}{|c|c|c|}
\hline$z_{p}$ (in $\left.\mathrm{mm}\right)$ & $u^{\prime \prime}$ & $v^{\prime \prime}$ \\
\hline \hline 0.6 & 0.480 & 0.335 \\
\hline 1.1 & 0.429 & 0.323 \\
\hline 1.8 & 0.445 & 0.308 \\
\hline 2.8 & 0.340 & 0.189 \\
\hline 3.6 & 0.156 & 0.105 \\
\hline
\end{tabular}

(b)

\begin{tabular}{|c|c|c|}
\hline$z_{p}$ (in $\left.\mathrm{mm}\right)$ & $u^{\prime \prime}$ & $v^{\prime \prime}$ \\
\hline \hline 0.6 & 0.215 & 0.145 \\
\hline 1.1 & 0.217 & 0.143 \\
\hline 1.8 & 0.221 & 0.134 \\
\hline 2.8 & 0.187 & 0.099 \\
\hline 3.6 & 0.128 & 0.069 \\
\hline
\end{tabular}

Table 1 Summary of $u^{\prime \prime}$ and $v^{\prime \prime}$ at five different X-Y measurement planes for (a) baseline, and (b) serrated trailing edge cases

In terms of $u^{\prime \prime}$, serrated aerofoil demonstrates values that are reduced by a half in comparison to the baseline case and this persists across $\mathrm{X}-\mathrm{Y}$ planes measured up until $z_{p}=2.8 \mathrm{~mm}$. Due to the gradual diminishing of turbulence activity when moving away from the wall (increasing $z_{p}$ ), the values of $u^{\prime \prime}$ decrease accordingly in both trailing edge cases.

More meaningful in terms of noise radiation, however, are velocity fluctuations in the wall-normal component $v^{\prime}$. Similarly to $u^{\prime \prime}, v^{\prime \prime}$ is decreased by more than a half in planes measured up to $z_{p}=2.8 \mathrm{~mm}$. Considering that the values at $z_{p}=3.6 \mathrm{~mm}$ for both trailing edge cases are closer to converging, this might indicate the extent of the effect that the flow regime responsible for these reductions in $u^{\prime \prime}$ and $v^{\prime \prime}$ has on the flow in the near vicinity of trailing edge serrations. The physical significance of the overall reduction in $v^{\prime} / U_{\infty}$ is related to the expression for the TNO-Blake model for near field unsteady surface pressure wavenumber frequency spectrum in Eq. 1. In other words, the far-field acoustic spectrum is affected by the $v$ velocity component at the trailing edge. It is therefore believed that this reduction in $v^{\prime}$ is one of the main constituents of the previously identified noise reduction that was originally demonstrated in Fig. 9.

At time $t_{3}$, baseline aerofoil still generates a detectable level of acoustic pressure (refer to Fig. 9a) and this is related to presence of relatively high level fluctuations $v^{\prime} / U_{\infty}$ at the edge where they can be scattered into noise. In the serrated trailing edge case, however, noise radiation associated with the SBL is almost completely diminished (see Fig. 9b). This correlates well with the corresponding $v^{\prime} / U_{\infty}$ contour in Fig. 13e and 13f, where no significant level of wall-normal velocity fluctuations is discernable at the oblique edges, meaning that only low level of noise radiation is expected. This provides further indication of the effect that serrations have on the flow in the near vicinity of trailing edge serrations, although a further and more conclusive investigation will follow. 

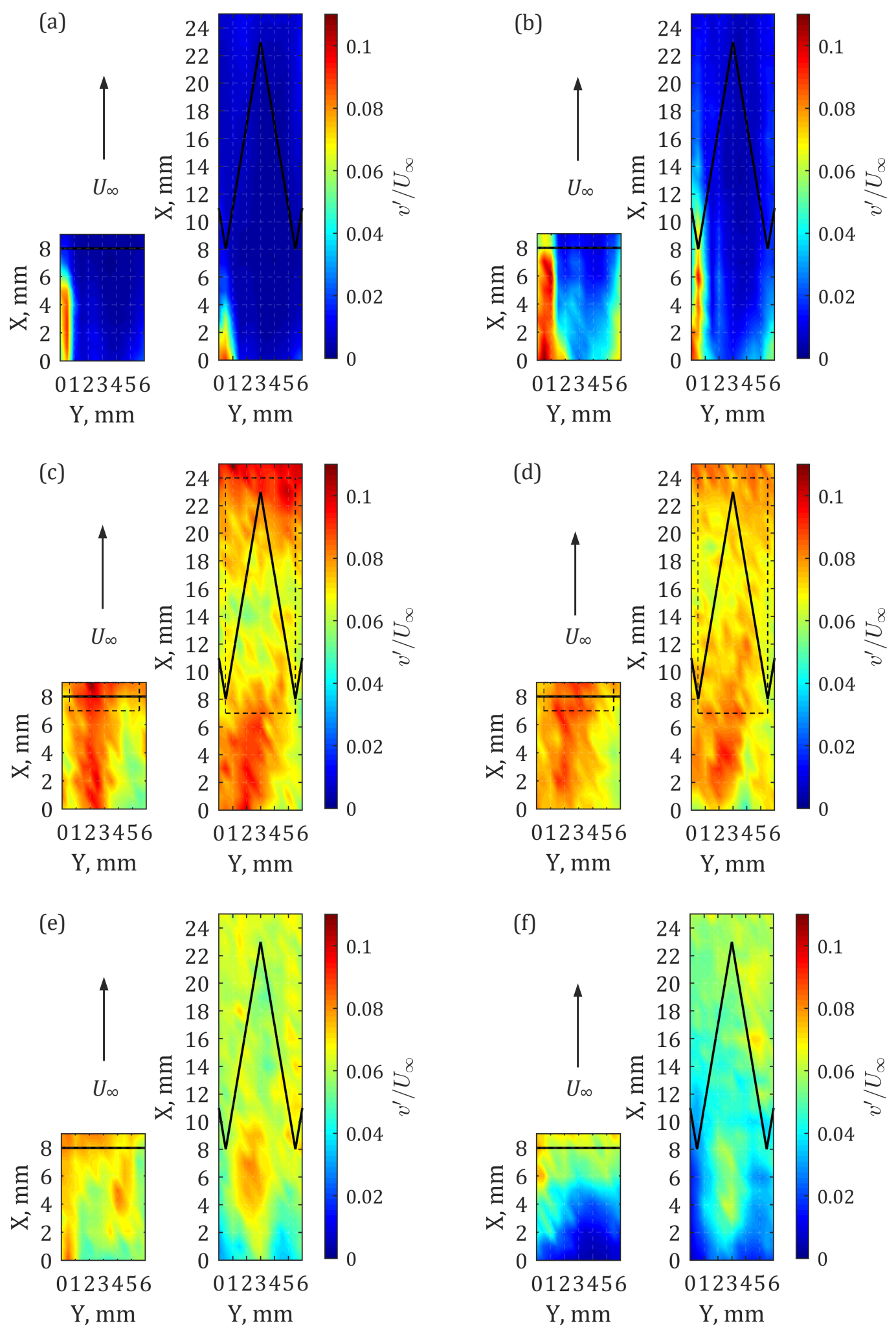

Fig. 13 Contour maps of SBL induced wall-normal velocity component fluctuations $v^{\prime} / U_{\infty}$ in the X-Y plane located at (a,c,e) $z_{p}=0.6 \mathrm{~mm}$ from the surface, and $(b, d, f) z_{p}=1.1 \mathrm{~mm}$ from the surface at three time

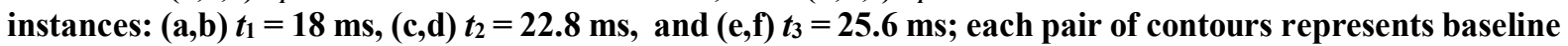
(Left) and serrated (Right) trailing edges 


\section{Formation of secondary flow in the near wake of serrations}

Flow development in the near wake region is studied by collecting data of $u$ (streamwise), $v$ (wall-normal) and $w$ (spanwise) velocity component fluctuations across Y-Z plane located at $0.5 \mathrm{~mm}$ from the trailing edge tip of the baseline aerofoil, and also at $0.5 \mathrm{~mm}$ from the trailing edge tip of serrated aerofoil. Note that the measurement planes for the baseline and serrated trailing edges are displaced by $15 \mathrm{~mm}$ (or by the length of the serration amplitude) due to serrations being added to the baseline aerofoil as shown in Fig. 2. Therefore, contour plots are presented at different instances of non-dimensional time, which is given by:

$$
t^{\prime}=\frac{t U_{\infty}}{C^{\prime}}
$$

where $t$ is time, $U_{\infty}$ is the velocity of the freestream flow and $C^{\prime}$ is the effective chord length of the aerofoil.

The following results are presented at three non-dimensional time instances $t_{1}^{\prime}, t_{2}^{\prime}$, and $t_{3}^{\prime}$. Noise radiation associated with these three non-dimensional time instances are displayed in Fig. 14.
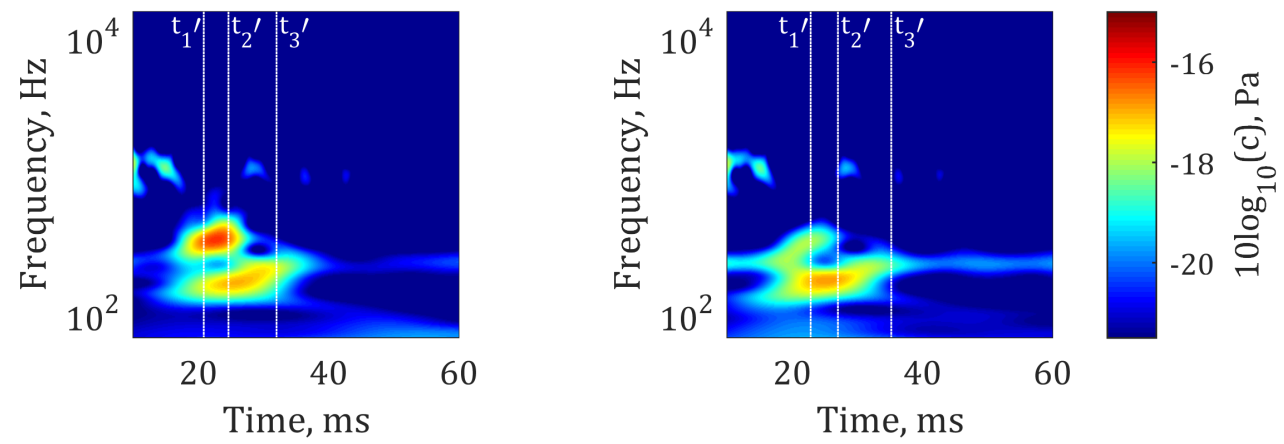

Fig. 14 Occurrence of non-dimensional time instances $t^{\prime}{ }_{1}, t^{\prime}{ }_{2}$ and $t^{\prime}{ }_{3}$ in relation to radiated noise

The results of measurements in the wake of the baseline aerofoil does not indicate any distinctive flow features in terms of both streamwise $u$ and wall-normal $v$ velocity components. Contour maps shown in Fig. 15 denote velocity perturbations $\tilde{V} / U_{\infty}$ of the $v$ component caused by the passage of the SBL through the Y-Z measurement plane. Generally, the overall structure of the SBL at $t^{\prime} 1$ is that of a turbulent boundary layer without secondary flow features. This also holds true for results presented at $t_{2}^{\prime}$ and $t^{\prime}{ }_{3}$. Data for the spanwise $w$ velocity component has not been collected under the assumption that the baseline trailing edge would not induce any significant spanwise velocity variations.
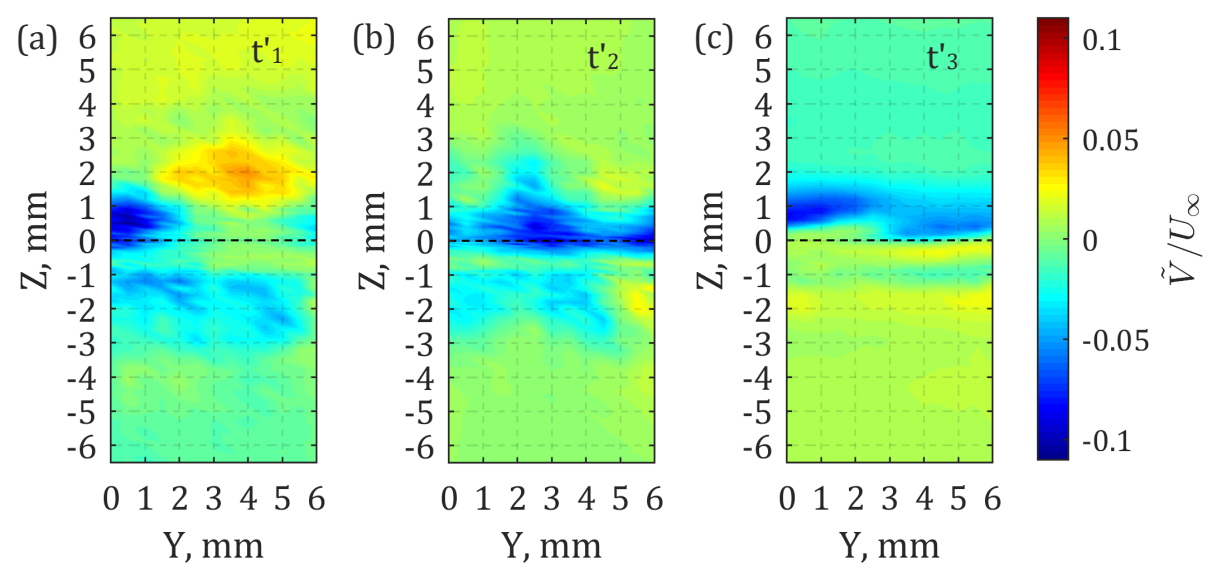

Fig. 15 Contour maps of SBL induced $\widetilde{V} / U_{\infty}$ in the $Y-Z$ plane located in the near wake, $0.5 \mathrm{~mm}$ from the baseline trailing edge tip; presented at three non-dimensional time instances (a) $t^{\prime} 1$, (b) $t^{\prime}$, and (c) $t^{\prime}{ }_{3}$. (---) - aerofoil trailing edge 

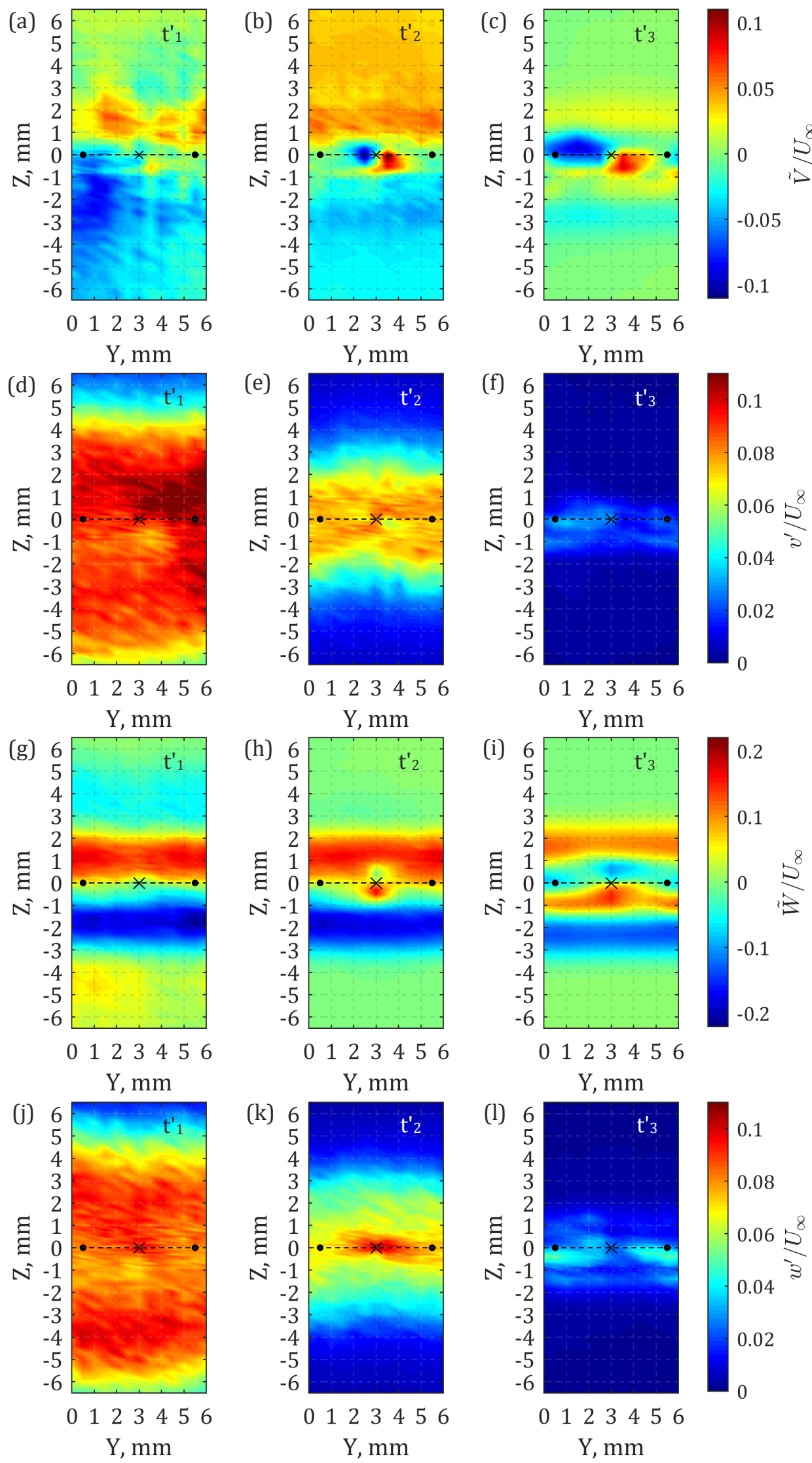

Fig. 16 Contour maps of SBL induced (a,b,c) $\widetilde{V} / U_{\infty},(\mathrm{d}, \mathrm{e}, \mathrm{f}) \boldsymbol{v}^{\prime} / U_{\infty},(\mathrm{g}, \mathrm{h}, \mathrm{i}) \widetilde{W} / U_{\infty}$, and (j,k,l) $w^{\prime} / U_{\infty}$ in the Y-Z plane located in the near wake, $0.5 \mathrm{~mm}$ from the serrated trailing edge tip; presented at three nondimensional time instances $t^{\prime}, t^{\prime} 2$ and $t^{\prime} 3_{2}(\times)$ - serration tip, $(\bullet)$ - serration root 
Contrary to the baseline case, measurement data collected in the wake of the serrated trailing edge provides interesting observations, more specifically to the flow structures induced by the serration. Results are similarly presented at the three non-dimensional time instances $t^{\prime}{ }_{1}, t_{2}^{\prime}$, and $t^{\prime}{ }_{3}$ in terms of wall-normal velocity component perturbations $\tilde{V} / U_{\infty}$ and rms fluctuations $v^{\prime} / U_{\infty}$ as well as the spanwise velocity component perturbations $\widetilde{W} / U_{\infty}$ and rms fluctuations $w^{\prime} / U_{\infty}$. At $t^{\prime}$, which corresponds to the time instance where noise (although at a much reduced level) is still radiated by the serrated trailing edge (see Fig. 14), all four contours indicate the arrival of the SBL and at this instance the flow still demonstrates typical synthetic turbulent boundary layer features.

Further development of the SBL indicates distinct structures forming around the serration tip that reach their fully developed state at instance $t^{\prime} 2$. Contour of $\tilde{V} / U_{\infty}$ (Fig. 16b) indicates two regions each side of serration tip one of positive and one of negative perturbation at $Y=2.5 \mathrm{~mm}$ and $Y=3.5 \mathrm{~mm}$ respectively. Similarly, contour of $\widetilde{W} / U_{\infty}$ (Fig. 16h) identifies two regions of spanwise flow motion - positive and negative perturbations at $Z=$ $0.5 \mathrm{~mm}$ and $Z=-0.5 \mathrm{~mm}$ respectively. This combination of flow movement in the $v$ and $w$ directions gives rise to a secondary flow in the form of distinct high momentum vortical structure with counter-clockwise rotation that is created around the serration tip. The structure is more clearly demonstrated by the mean velocity vector plot in Fig. 17b, obtained from the $v$ and $w$ component measurement data.

In conjunction with the vortical structure formed around the serration tip, the contour map of $v^{\prime} / U_{\infty}$ fluctuations shown in Fig. 16e presents two areas of reduced turbulence intensity each side of the tip, coinciding with the two regions of significant momentum variation identified previously in Fig. 16b. At this particular instance, however, the aforementioned low turbulence intensity structures are not very distinctive, although their persistence can be identified at 4 consecutive time instances (see Fig. 18 in the Appendix) that occur prior to $t^{\prime}$. This reduction in $v^{\prime} / U_{\infty}$ is simultaneously accompanied by a high concentration of spanwise fluctuations $w^{\prime} / U_{\infty}$ around the serration tip as illustrated in Fig. 16k. The significance of this observation is the indication of a mechanism by which wall-normal velocity fluctuations have given way to an increase in fluctuations of the spanwise velocity component $w^{\prime} / U_{\infty}$. The latter have significantly less contribution to the acoustic scattering in comparison to $v^{\prime} / U_{\infty}$. The presence of such reduction in $v^{\prime} / U_{\infty}$ in the near wake could implicate the weakened mechanism of noise scattering at the serration edges. To investigate this further, the parameters at nondimensionalised time instance $t_{1}^{\prime}$ are again considered. At this instance, the vortical structure has not yet formed in the wake, implying that it must still be in the development stage somewhere upstream of the serration tip. This time instance can actually be referred back to the results presented previously in section E.2, where both the largest reduction in the radiated acoustic pressure and the reduction in $v^{\prime} / U_{\infty}$ over the serration surface were shown to occur at $t_{2}=22.8 \mathrm{~ms}$. In terms of absolute time, $t_{1}^{\prime}$ and $t_{2}$ in fact represent the exact same time instance. At this time instance, the vortical structure that was later seen in the wake at $t^{\prime} 2$ is already present at the serration surface, or the serration edges as previously proposed in ${ }^{12,14}$. Assuming that this secondary flow structure maintains the ability to reduce the transverse velocity component fluctuations $v^{\prime} / U_{\infty}$ as described earlier, the intensity of turbulence interaction with the serration edge would be weakened, leading to diminished scattering of the hydrodynamic pressure waves at the serration edges. This could indicate as one of the mechanisms that is responsible for the momentary reduction in radiated acoustic pressure that was observed previously. This will also explain the reduction in $v^{\prime} / U_{\infty}$ described in section E.2. Note that the physical dimensions of the cross-wire probe have imposed a limitation on the measurement point nearest to the trailing edge surface being at least $0.6 \mathrm{~mm}$ from it. Considering that in terms of $\tilde{V} / U_{\infty}$ the vertical extent of the vortex that is seen around the tip at its fully developed state is only approximately $0.5 \mathrm{~mm}$, the upstream development of this structure, if any, could not be captured experimentally. At a later instance, $t_{3}^{\prime}$, the stable vortical structure begins to disintegrate, redistributing and giving momentum away to the bulk of the SBL.

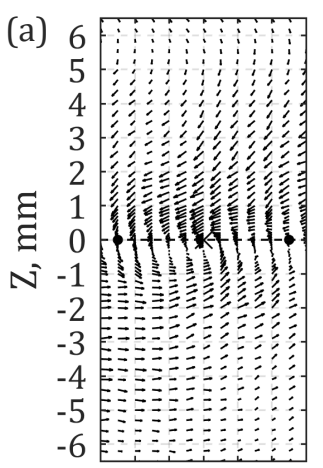

0123456 $\mathrm{Y}, \mathrm{mm}$

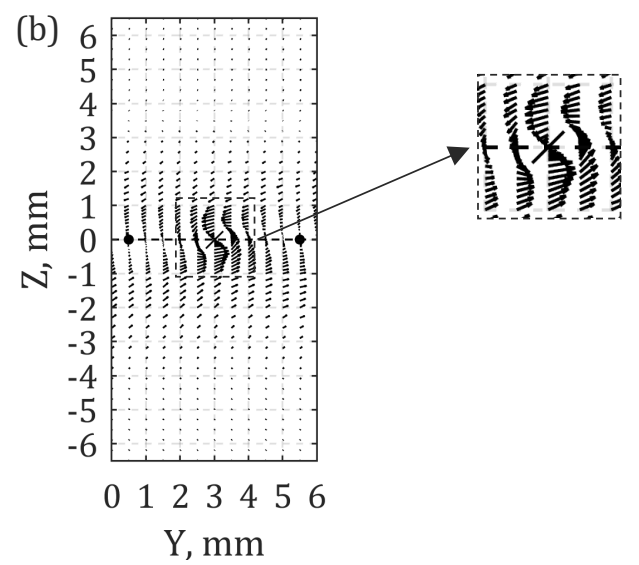

Fig. 17 Mean velocity vectors in the wake of the serrated trailing edge at non-dimensional time instances of (a) $t^{\prime}$, and (b) $t^{\prime}$..$(\times)$ - serration tip, $(\bullet)$ - serration root 


\section{V.Conclusion}

The temporal-spatial development of interaction between a synthetically induced turbulent boundary layer, and a straight or serrated aerofoil trailing edge in low Reynolds number flow, was investigated experimentally in the current study. The experiments were conducted at a Reynolds number of $\operatorname{Re}=0.96 \times 10^{5}$ on a NACA 0008 aerofoil placed at an effective angle of attack of $0^{\circ}$. Hot-wire measurements of three velocity components were performed in the near vicinity of trailing edge surface and near wake simultaneously with far-field noise measurements. Ensemble phase-averaged hot wire data has enabled the reconstruction of the spatial and temporal development of the synthetic turbulent boundary layer structure as it interacts with the straight or serrated trailing edge. The temporal variation in trailing edge self-noise radiated due to this interaction is presented after performing a wavelet analysis of the acoustic data.

At the experimental conditions selected for this study, no significant changes to the boundary layer upstream of the serrations are observed. This goes in line with the observations made in previous studies. However, at the regions coinciding with the serration surface, and also in the near wake, the flow undergoes significant changes with the presence of time dependent secondary flow. At the time instance that correlates to the largest level of noise reduction by the serration, hot-wire measurements in several planes measured parallel to the serration surface indicated a reduction in the wall-normal velocity component fluctuations. Similar reductions are also identified in the wake region and this is associated with a high momentum counter - clockwise rotating vortical structure that forms around the serration tip as observed in the near wake. This vortical structure can redistribute the turbulence level from a transverse direction to a spanwise direction, thereby increasing the anisotropic level of the turbulence at the serration. The results presented thus far suggest that before reaching the wake region, such vortical structures exist at locations coinciding with the serration edges. This would explain the reduction in fluctuations in the planes measured parallel to the serration surface. Their existence would diminish the intensity of turbulence interaction with the serration surface and the edges, consequently reducing the level of scattering that occurs. This represents one of the mechanisms by which serrations can reduce noise. A more in-depth data analysis in support of this observation is currently underway. Hot-wire measurement data of the transverse and streamwise velocity components should allow conducting a quadrant analysis using the information of the synthetic turbulent boundary layer induced velocity fluctuations. This will shed more light on the mechanism of turbulence production at the serration surface as well as in the near wake.

\section{Appendix}
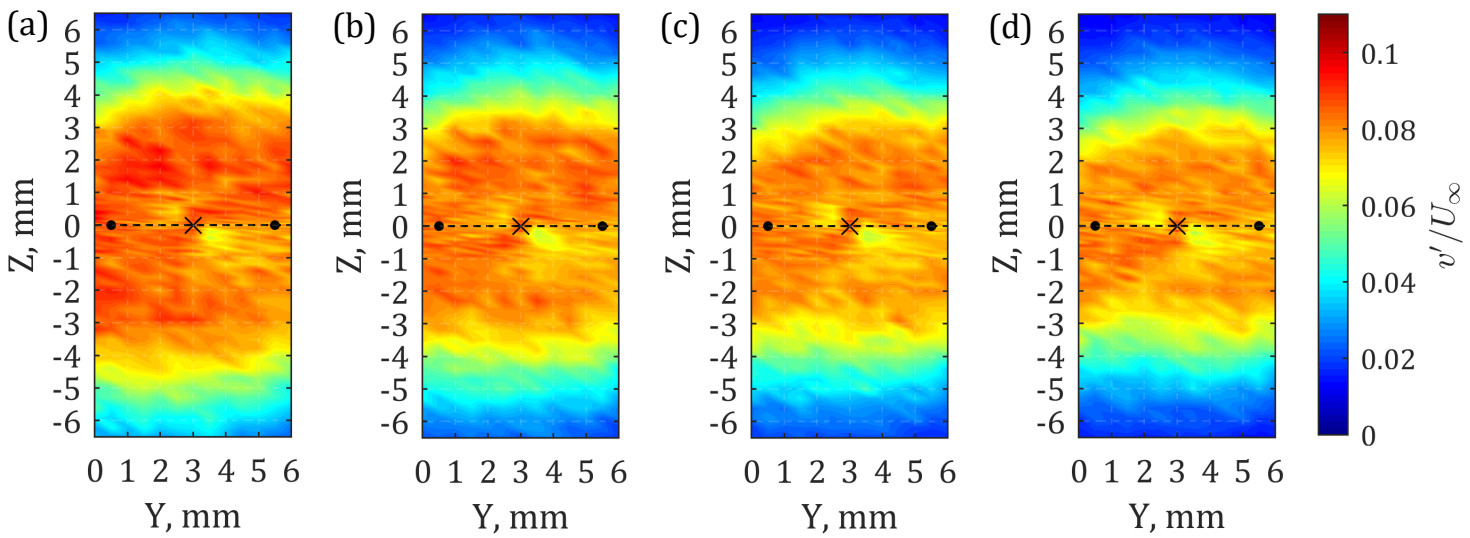

Fig. 18 Contour maps of SBL induced $v^{\prime} / U_{\infty}$ in the Y-Z plane located in the near wake, $0.5 \mathrm{~mm}$ from the baseline trailing edge tip; presented at four consecutive non-dimensional time instances. $(\times)-$ serration tip, $(\bullet)$ - serration root.

\section{Acknowledgments}

The authors would like to thank for the financial support from the EPSRC Doctoral Training Partnership (DTP), and the EPSRC Research Grant No: EP/N018737/1 on the "Quiet Aerofoils of the Next Generation".

\section{References}


Oerlemans, S., Fisher, M., Maeder, T., and Kögler, K., "Reduction of Wind Turbine Noise Using Optimized Airfoils and Trailing-Edge Serrations," AIAA Journal, vol. 47, 2009, pp. 1470-1481.

Brooks, T. F., Pope, S., and Marcolini, M. A., "Airfoil Self-Noise and Prediction,” NASA Reference Publication 1218, 1989, pp. 1-142.

Oerlemans, S., "Reduction of wind turbine noise using blade trailing edge devices," 22nd AIAA/CEAS Aeroacoustics Conference, Lyon, France: 2016, pp. 1-18.

Braun, K., Van der Borg, N., Dassen, A., Doorenspleet, F., Gordner, A., Ocker, J., and Parchen, R., Serrated trailing edge noise (STENO), 1999.

Chong, T. P., and Joseph, P. F., "An experimental study of airfoil instability tonal noise with trailing edge serrations," Journal of Sound and Vibration, vol. 332, 2013, pp. 6335-6358.

Chong, T. P., Vathylakis, A., Joseph, P., and Gruber, M., "On the Noise and Wake flow of an Airfoil with Broken and Serrated Trailing Edges," 17th AIAA/CEAS Aeroacoustics Conference, Portland, Oregon: 2011, pp. 1-19.

Dassen, T., Parchen, R., Bruggeman, J., and Hagg, F., "Results of a wind tunnel study on the reduction of airfoil selfnoise by the application of serrated blade trailing edges," European Union Wind Energy Conference and Exhibition, Göteborg, Sweden: 1996, pp. 800-803.

Zhu, W., and Shen, W., “Airfoil Trailing Edge Noise Generation and Its Surface Pressure Fluctuation,” Journal of Power and Energy Engineering, vol. 3, 2015, pp. 14-19.

Howe, M. S., "Noise produced by a sawtooth trailing edge," The Journal of the Acoustical Society of America, vol. 90, 1991, pp. 482-487.

Gruber, M., Joseph, P., and Chong, T. P., "Experimental Investigation of Airfoil Self Noise and Turbulent Wake Reduction by the use of Trailing Edge Serrations," 16th AIAA/CEAS Aeroacoustics Conference, Stockholm, Sweden: 2010, pp. 1-23.

Chong, T. P., and Vathylakis, A., "On the aeroacoustic and flow structures developed on a flat plate with a serrated sawtooth trailing edge," Journal of Sound and Vibration, vol. 354, 2015, pp. 65-90.

Gruber, M., "Airfoil noise reduction by edge treatments," Ph.D. Dissertation, Faculty of Engineering and the Environment, Institute of Sound and Vibration, University of Southampton, UK, 2012.

Avallone, F., Pröbsting, S., and Ragni, D., "Three-dimensional flow field over a trailing-edge serration and implications on broadband noise," Physics of Fluids, vol. 28, 2016, pp. 1-20.

Van der Velden, W. C., Avallone, F., and Ragni, D., "Numerical analysis of noise reduction mechanisms of serrated trailing edges under zero lift condition," 23rd AIAA/CEAS Aeroacoustics Conference, Denver, Colorado: 2017, pp. $1-14$.

Amiet, R. K., "Noise due to turbulent flow past a trailing edge," Journal of Sound and Vibration, vol. 47, 1976, pp. $387-393$.

Parchen, R., Progress report DRAW: A prediction scheme for trailing-edge noise based on detailed boundary-layer characteristics, TNO Rept. HAGRPT-980023, TNO Institute of Applied Physics, The Netherlands: 1998.

Schubauer, G. B., and Klebanoff, P. S., "Contributions on the mechanics of boundary-layer transition," NACA Technical Report 1289, 1955, pp. 853-863.

Corrsin, S., and Kistler, A. L., "Free-Stream Boudaries of Turbulent Flows," NACA Technical Report 1244, 1954, pp. 1-32.

Gad-El-Hak, M., Blackwelderf, R. F., and Riley, J. J., "On the growth of turbulent regions in laminar boundary layers," Journal of Fluid Mechanics, vol. 110, 1981, pp. 73-95.

Gostelow, J. P., Melwani, N., and Walker, G. J., "Effects of Streamwise Pressure Gradient on Turbulent Spot Development," Journal of Turbomachinery, vol. 118, 1996, pp. 737-743. 
1999, p. 588.

Wygnanski, I., Sokolov, M., and Friedman, D., “On a turbulent 'spot' in a laminar boundary layer," Journal of Fluid Mechanics, vol. 78, 1976, pp. 785-819.

Perry, A. E., Lim, T. T., and Teh, E. W., “A visual study of turbulent spots,” Journal of Fluid Mechanics, vol. 104, 1981, p. 387.

Chambers, F. ., "Synthetically generated turbulent boundary layer development and structure," AIAA Shear Flow Control Conference, Boulder, Colorado: 1985, pp. 1-8.

Vathylakis, A., Chong, T.P., Kim, J. H., "Design of a low-noise aeroacoustic wind tunnel facility at Brunel University," 20th AIAA/CEAS Aeroacoustics Conference, Atlanta, Georgia: 2014, pp. 1-11.

Morton, M. A., Devenport, W. J., Ravetta, P. A., Borgoltz, A., Barone, M. F., Burdisso, R. A., and Brown, K. A., "The Kevlar-walled anechoic wind tunnel," Journal of Sound and Vibration, vol. 332, 2013, pp. 3971-3991.

Glezer, A., Katz, Y., and Wygnanski, I., "On the breakdown of the wave packet trailing a turbulent spot in a laminar boundary layer,” Journal of Fluid Mechanics, vol. 198, 1989, pp. 1-26.

Wygnanski, I., "The Effects of Reynolds Number and Pressure Gradient on the Transitional Spot in a Laminar Boundary Layer," The Role of Coherent Structures in Modelling Turbulence and Mixing, 1981, pp. 304-332.

Chong, T. P., and Zhong, S., "On the Three-Dimensional Structure of Turbulent Spots," Journal of Turbomachinery, vol. 127, 2005, p. 545 